\title{
Diabetes Distress or Major Depressive Disorder? A Practical Approach to Diagnosing and Treating Psychological Comorbidities of Diabetes
}

\author{
Kathryn Evans Kreider
}

Received: November 30, 2016 / Published online: February 3, 2017

(C) The Author(s) 2017. This article is published with open access at Springerlink.com

\begin{abstract}
The presence of major depressive disorder (MDD) in people with diabetes may be up to three times more common than in the general population. People with diabetes and major depressive disorder have worse health outcomes and higher mortality rates. Diabetes distress refers to an emotional state where people experience feelings such as stress, guilt, or denial that arise from living with diabetes and the burden of self-management. Diabetes distress has also been linked to worse health outcomes. There are multiple treatment options for MDD including pharmacotherapy and cognitive behavioral approaches. Providers treating patients with diabetes must be aware of the frequent comorbidity of diabetes, diabetes distress, and depression and manage patients using a multidisciplinary team approach. This article discusses the epidemiology, pathophysiology, and bi-directional relationship of diabetes and depression and provides a practical,
\end{abstract}

Enhanced content To view enhanced content for this article go to http://www.medengine.com/Redeem/ 7687F0601977C0A2.

K. E. Kreider $(\varangle)$

Duke University School of Nursing, Durham,

NC, USA

e-mail: kathryn.evans@duke.edu

K. E. Kreider

Duke University Medical Center, Durham, USA patient-centered approach to diagnosis and management.

Keywords: Chronic care management; Depression; Diabetes distress; Major depressive disorder

\section{INTRODUCTION}

Major depressive disorder (MDD) is a debilitating chronic condition that may affect every aspect of life. Studies have shown that people with diabetes and MDD are more likely to have poorer health outcomes including uncontrolled blood sugars, complications from diabetes, and higher rates of all-cause mortality [1]. In addition to the high comorbidity of depression and diabetes, a new descriptive term has emerged called diabetes distress, defined as an emotional state that causes significant emotional distress but does not meet the qualifications for MDD. Diabetes distress has also been shown to negatively affect health outcomes. The 2017 American Diabetes Association Standards of Medical Care in Diabetes highlights the importance of screening, diagnosis, and management of both of these conditions to achieve optimal outcomes, including improved self-care, glycemic control, reduced cardiovascular risk, and reduced all-cause mortality. 


\section{EPIDEMIOLOGY}

Major depressive disorder is a complex, debilitating condition that affects millions of people worldwide. It is commonly recognized that diabetes and MDD frequently coexist. It is estimated that the prevalence of MDD in people with type 1 diabetes (T1DM) is nearly three times as high as in the general population (12\% vs 3\%) and nearly twice as high for those with type 2 diabetes than those without diabetes (19\% vs. 10\%) [2]. Significant depressive symptoms occur in approximately 1 in 4 people with T1DM or T2DM [3]. Women are affected at a higher rate [2]. Other risk factors include those diagnosed with diabetes at a young age, high body mass index (BMI), poor glycemic control, complications of diabetes, and low levels of education [4]. The comorbidity of MDD and diabetes exists across race and cultures [5]. Many experts suggest that the prognosis of MDD and diabetes is worse when the conditions coexist than when they occur independently [6].

It is also important to recognize that patients may suffer from diabetes distress, a term that describes significant emotional reactions to the diagnosis, threat of complications, self-management demands, or unsupportive social structures surrounding diabetes [7]. Up to $45 \%$ of people in community settings with T2DM may suffer from diabetes distress [7] and approximately $70 \%$ of those individuals do not meet the criteria for MDD [8]. Diabetes distress is also linked to suboptimal glycemic control and decreased self-management behaviors [9].

\section{PATHOPHYSIOLOGY}

Traditionally it was believed that the link between MDD and diabetes was related to the burden of chronic disease management [10]. We now understand that the relationship between diabetes and MDD is likely to be bi-directional and the causes are not entirely clear. There are several indications that MDD may contribute to the development of diabetes including hyperactivity of the hypothalamic-pituitary-adrenal (HPA) axis and sympathomedullary activation which can lead to decreased glucose transport and insulin resistance [1]. Further, MDD often causes reduced physical activity levels which can contribute to obesity, increased cortisol levels, and insulin resistance [1].

Diabetes may contribute to the onset of MDD due to patient burden of chronic disease management, requirements for high levels of self-care, and nervous system activation which has been associated with MDD [11]. Other factors may include perceived disability, low levels of psychological support [12], antidepressant medications, disrupted sleep, or environmental factors, all of which may contribute to insulin resistance [10]. Further, there are several overlapping pathophysiologic processes between MDD and diabetes including oxidative stress, decreased glucose utilization, glucocorticoid signaling, and inflammatory processes [13].

\section{CLINICAL CONSEQUENCES OF UNTREATED MDD IN DIABETES}

There are significant clinical consequences to untreated MDD and emotional distress in diabetes. A meta-analysis of over 16,000 participants suggested that people with diabetes with depression of any severity are less likely to engage in appropriate self-care behaviors (such as physical activity and smoking cessation) and participate in quality care such as annual eye exams and immunizations [14], leading to an increase in subsequent complications. Additionally, quality of life is impaired for those with MDD and diabetes, including aspects such as physical function, social function, and overall health [15].

A prospective study of 4623 patients with T2DM suggested that MDD was significantly associated with increased risk of microvascular (hazard ratio 1.36 [95\% CI 1.05-1.75]) and macrovascular (1.24 [1.0-1.54]) outcomes, even after adjusting for diabetes severity and self-care behaviors [16]. The ACCORD trial (Action to Control Cardiovascular Risk in Diabetes) of 1028 substudy participants suggested that MDD was an independent risk factor for all-cause mortality after adjusting for blood pressure, A1c, lipids, BMI, aspirin use, tobacco, alcohol, 
living alone, and education level [17]. Other systematic reviews and meta-analyses have similarly suggested that MDD in people with diabetes leads to higher all-cause mortality [18].

The relationship between MDD and A1c is not clear. Some studies suggest a deterioration of A1c associated with MDD [19] while other studies show an inconsistent relationship [20]. Diabetes distress has been shown to positively correlate with A1c levels [8,9] and negatively correlate with physical activity levels [9].

All studies considered, it is clear that the presence of MDD has an inverse relationship with optimal diabetes outcomes.

\section{CLINICAL PRESENTATION AND DIAGNOSIS}

The American Diabetes Association recommends that screening for MDD should occur annually, when complications are diagnosed, if a change in medical status occurs, or if there are significant changes in life circumstances [21]. In order to be diagnosed with MDD, patients must present with five or more symptoms that occur over a 2-week period and represent a significant change from baseline functioning. The symptoms of diabetes distress may present similarly to MDD but lack the severity to qualify as MDD. Symptoms that may occur in MDD and diabetes distress appear in Table 1. In people with diabetes, some of the symptoms may be attributed to uncontrolled blood sugars and should be evaluated carefully. For example, it is common that patients with hyperglycemia experience fatigue and weight loss. Similarly, patients with frequent hypoglycemia may experience insomnia and diminished ability to concentrate. Correlating symptoms with glycemic levels should be included as part of the diagnostic process. Thyroid disorders, obstructive sleep apnea, obesity, and vitamin deficiencies are common in patients with diabetes and should be ruled out before the diagnosis and treatment for MDD commences.

Table 1 Symptoms of MDD and diabetes distress [23, 37]

\begin{tabular}{|c|c|}
\hline Symptoms of major depressive disorder & Symptoms of diabetes distress \\
\hline $\begin{array}{l}\text { Five or more symptoms must be present over at least } 2 \text { weeks and represent } \\
\text { significant change from baseline functioning }\end{array}$ & $\begin{array}{l}\text { Emotional response varies across individuals } \\
\text { and may include feeling }\end{array}$ \\
\hline Patients must exhibit either anhedonia or depressed mood & Unmotivated \\
\hline Other symptoms may include & Burned out \\
\hline Diminished interest/pleasure in daily activities & Overwhelmed \\
\hline Appetite changes (weight loss/gain) & Frustrated \\
\hline Insomnia or hypersomnia & Defeated \\
\hline Psychomotor agitation or retardation & Angry \\
\hline Fatigue & Guilt \\
\hline Feelings of worthlessness or guilt & Denial \\
\hline \multirow[t]{5}{*}{ Diminished concentration ability } & Fear (of hypoglycemia or complications) \\
\hline & Lonely \\
\hline & Actions may include \\
\hline & Poor self-care behaviors \\
\hline & Lack of adherence to diabetes regimen \\
\hline
\end{tabular}


In a primary care setting, there are several widely available validated tools to assist with the diagnosis of MDD including the Primary Care Health Questionnaire (PHQ-9), Beck Depression Inventory (BDI-II), or the Hospital Anxiety and Depression Scale (HADS) [22]. The most widely used scale is the PHQ-9, a 9-question survey completed by the patient to help identify those at risk for MDD. Some authors suggest that a more straightforward approach asking two questions (PHQ-2) can help identify those at risk for MDD including:

- "During the past month, have you been bothered by having little interest or pleasure in doing things?"

- "During the past month, have you been bothered by feeling down, depressed, or hopeless?" [10].

If the answer to either of these questions is "yes" then options should be explored for further diagnosis and management if the patient is willing.

Diabetes distress can be evaluated using the Problem Areas in Diabetes Survey (PAID), a 20 -question validated survey assessing various areas of psychological stress related to diabetes [23]. Shorter surveys have been developed including the PAID-5 (sensitivity 94\%, specificity 89\%) and the PAID-1 (sensitivity and specificity $\sim 80 \%$ ) [24].

\section{MANAGEMENT}

Traditional therapies for MDD include medication and psychotherapy. Classes of these medications include selective serotonin reuptake inhibitors (SSRIs), tricyclics, and serotonin-norepinephrine reuptake inhibitors (SNRIs). SSRIs are the preferred pharmacologic therapy owing to their safety profile and efficacy. All SSRIs seem to be equally efficacious at reducing depressive symptoms at appropriate doses [25]. Medications should be selected on the basis of side effect profiles, patient preferences, and individual response to treatment [26]. Other medications to treat MDD such as tricyclic antidepressants (TCAs) and monoamine oxidase inhibitors (MAOIs) are rarely recommended for patients with diabetes because of unfavorable side effects such as increased weight gain, increased insulin resistance, and potential cardiotoxicity. There is some data that sertraline [27] and fluoxetine [28] may have a slight benefit to glycemic control but these results have not been widely replicated. There is also some data that bupropion may induce weight loss in people with diabetes and MDD, potentially leading to improved cardiovascular risk profile [29].

Cognitive behavioral therapy (CBT), a method of reframing negative thoughts, is a widely documented, empirically validated type of psychotherapy. Some studies suggest that CBT can improve medication adherence, depressive symptoms, and glycemic control in patients with T2DM [30]. Other approaches include interpersonal therapy, motivational interviewing, psychodynamic therapy, and Web-based therapeutic approaches [10]. Diabetes self-management education (DSME) is an effective treatment for diabetes distress [31]. It is important to note that most of the studies include people with T2DM and there is a more limited knowledge base about how psychologically based interventions affect people with T1DM [10].

A "watchful waiting" approach to treating mild depressive symptoms may be plausible since up to $40 \%$ of patients will improve without any intervention [4]; this may include observing the patient for 8-12 weeks for symptom changes while encouraging healthy lifestyle approaches to managing symptoms of MDD. Patients who have diabetes distress are unlikely to benefit from pharmacotherapy [32].

A referral to a mental health care provider may be necessary in certain situations. These circumstances may include suspicion of a serious mental illness, ongoing self-care impairment after individualized patient education, ongoing disordered eating behavior, or repeated hospitalizations for diabetic ketoacidosis [21].

Collaborative care amongst primary care physicians, nurses, and other professionals has been shown to improve MDD remission and higher adherence to diabetes and MDD medications [10, 33]. A 2016 randomized controlled trial suggested that "patient prioritized planning" that incorporated knowledge of the individual patient's financial, social, and emotional 
needs significantly improved A1c and MDD symptoms, compared to more basic MDD counseling [34]. Interestingly, a 2016 study with 3609 patients used a team-based approach based on the chronic care model and suggested that $24 \%$ of people with diabetes and MDD experienced remission of their MDD while only $16 \%$ showed improvement in their depressive symptoms over 11 months [35]. These numbers suggest that MDD and diabetes can be very difficult to treat, even with targeted, individualized management approaches. All clinicians should consider incorporating aspects of good clinical care into encounters with patients with diabetes such as attentive and empathetic listening, sensitive inquiry, and the use of reflective observations [36].

\section{SUMMARY}

Both MDD and diabetes distress are commonly associated with diabetes and can significantly affect health outcomes. Clinicians should be aware of the common coexistence of MDD and diabetes distress in people with diabetes and be prepared to diagnose and manage these conditions as a part of routine diabetes management using a team-based approach [35].

\section{ACKNOWLEDGEMENTS}

No funding was received for publication of this article. The named author meets the International Committee of Medical Journal Editors (ICMJE) criteria for authorship for this manuscript, takes responsibility for the integrity of the work as a whole, and has given final approval for the version to be published.

Disclosures. Kathryn Evans Kreider has nothing to disclose.

Compliance with Ethics Guidelines. This article does not contain any new studies with human or animal subjects performed by the author.
Open Access. This article is distributed under the terms of the Creative Commons Attribution-NonCommercial 4.0 International License (http://creativecommons.org/licenses/ by-nc/4.0/), which permits any noncommercial use, distribution, and reproduction in any medium, provided you give appropriate credit to the original author(s) and the source, provide a link to the Creative Commons license, and indicate if changes were made.

\section{REFERENCES}

1. Rustad JK, Musselman DL, Nemeroff CB. The relationship of depression and diabetes: pathophysiological and treatment implications. Psychoneuroendocrinology. 2011;36(9):1276-86.

2. Roy T, Lloyd CE. Epidemiology of depression and diabetes: a systematic review. J Affect Disord. 2012;142:S8-21.

3. Anderson RJ, Freedland KE, Clouse RE, Lustman PJ. The prevalence of comorbid depression in adults with diabetes: a meta-analysis. Diabetes Care. 2001;24:1069-78.

4. Hermanns N, Caputo S, Dzida G, Khunti K, Meneghini LF, Snoek F. Screening, evaluation and management of depression in people with diabetes in primary care. Primary Care Diabetes. 2013;7(1):1-10.

5. Lloyd CE, Roy T, Nouwen A, Chauhan AM. Epidemiology of depression in diabetes: international and cross-cultural issues. J Affective Disord. 2012;142:S22-29.

6. Lloyd CE, Pambianco G, Orchard TJ. Does diabetes-related distress explain the presence of depressive symptoms and/or poor self-care in individuals with type 1 diabetes? Diabet Med. 2010;27(2):234-7.

7. Fisher L, Hessler DM, Polonsky WH, Mullan JT. When is diabetes distress clinically meaningful? Establishing cut points for the Diabetes Distress Scale. Diabetes Care. 2012;35:259-64.

8. Fisher L, Mullan JT, Arean P, Glasgow RE, Hessler D, Masharani U. Diabetes distress but not clinical depression or depressive symptoms is associated with glycemic control in both cross-sectional and longitudinal analyses. Diabetes Care. 2010;33:23-8. 
9. Fisher L, Glasgow RE, Strycker LA. The relationship between diabetes distress and clinical depression with glycemic control among patients with type 2 diabetes. Diabetes Care. 2010;33(5):1034-6.

10. Holt RI, De Groot M, Golden SH. Diabetes and depression. Curr Diab Rep. 2014;14(6):1-9.

11. Knol MJ, Heerdink ER, Egberts AC, et al. Depressive symptoms in subjects with diagnosed and undiagnosed type 2 diabetes. Psychosom Med. 2007;69(4):300-305.

12. Talbot F, Nouwen A. A review of the relationship between depression and diabetes in adults: is there a link? Diabetes Care. 2000;23(10):1556-62.

13. McIntyre RS, Soczynska JK, Konarski JZ, et al. Should depressive syndromes be reclassified as "metabolic syndrome type II"? Ann Clin Psychiatry. 2007;19(4): 257-264.

14. Egede LE, Ellis C, Grubaugh AL. The effect of depression on self-care behaviors and quality of care in a national sample of adults with diabetes. Gen Hosp Psychiatry. 2009;31(5):422-7.

15. Schram MT, Baan CA, Pouwer F. MDD and quality of life in patients with diabetes: a systematic review from the European Depression in Diabetes (EDID) research consortium. Curr Diabetes Rev. 2009;5(2):112-9.

16. Lin EH, Rutter CM, Katon W, et al. Depression and advanced complications of diabetes a prospective cohort study. Diabetes Care. 2010;33(2):264-9.

17. O'Connor PJ, Narayan KM, Anderson R, et al. Effect of intensive vs standard blood pressure control on depression and health-related quality of life in type 2 diabetes: the ACCORD trial. Diabetes Care. 2012;35:1479-81.

18. van Dooren FE, Nefs G, Schram MT, Verhey FR, Denollet J, Pouwer F. Depression and risk of mortality in people with diabetes mellitus: a systematic review and meta-analysis. PLoS One. 2013;8(3): e57058.

19. Lustman PJ, Anderson RJ, Freedland KE, deGroot M, Carney RM, Clouse RE. Depression and poor glycemic control: a meta-analytic review of the literature. Diabetes Care. 2000;23:934-42.

20. Aikens JE, Perkins DW, Piette JD, Lipton B. Association between depression and concurrent type 2 diabetes outcomes varies by diabetes regimen. Diabet Med. 2008;25(11):1324-9.

21. American Diabetes Association. Standards of medical care in diabetes-2017. Diabetes Care. 2017;40(Suppl 1):S39-40.
22. Roy T, Lloyd CE, Pouwer F, Holt RI, Sartorius N. Screening tools used for measuring depression among people with type 1 and type 2 diabetes: a systematic review. Diabetes Med. 2012;29:164-75.

23. Polonsky WH, Anderson BJ, Lohrer PA, et al. Assessment of diabetes-related distress. Diabetes Care. 1995;18(6):754-60.

24. McGuire BE, et al. Short-form measures of diabetes-related emotional distress: the Problem Areas in Diabetes Scale (PAID)-5 and PAID-1. Diabetologia. 2010;53(1):66-9.

25. Markowitz SM, Gonzalez JS, Wilkinson JL, Safren SA. A review of treating depression in diabetes: emerging findings. Psychosomatics 2011;52(1):1-18.

26. National Collaborating Centre for Mental Health. Common mental health disorders: identification and pathways to care CG123. London: National Institute of Health and Clinical Excellence; 2011.

27. van der Feltz-Cornelis CM, Nuyen J, Stoop C, et al. Effect of interventions for major depressive disorder and significant depressive symptoms in patients with diabetes mellitus: a systematic review and meta-analysis. Gen Hosp Psychiatry. 2010;32: 380-95.

28. Ye Z, Chen L, Yang Z, et al. Metabolic effects of fluoxetine in adults with type 2 diabetes mellitus: a meta-analysis of randomized placebo-controlled trials. PLoS One. 2011;6(7):e21551.

29. Hollander P, Gupta AK, Plodkowski R, et al. Effects of naltrexone sustained-release/bupropion sustained-release combination therapy on body weight and glycemic parameters in overweight and obese patients with type 2 diabetes. Diabetes Care. 2013;36(12):4022-9.

30. Safren SA, Gonzalez JS, Wexler DJ, et al. A randomized controlled trial of cognitive behavioral therapy for adherence and depression (CBT-AD) in patients with uncontrolled type 2 diabetes. Diabetes Care. 2014;37(3):625-33.

31. Fisher L, Hessler D, Glasgow RE, et al. REDEEM: a pragmatic trial to reduce diabetes distress. Diabetes Care. 2013;36(9):2551-8.

32. Fournier JC, DeRubeis RJ, Hollon SD, et al. Antidepressant drug effects and depression severity: a patient-level meta-analysis. JAMA. 2010;303(1): 47-53.

33. Huang Y, Wei X, Wu T, Chen R, Guo A. Collaborative care for patients with depression and diabetes mellitus: a systematic review and meta-analysis. BMC Psychiatry. 2013;13(1):1. 
34. de Vries M, Heather F, Boyle KB, Rooney K, Bogner HR. Diabetes and depression care: a randomized controlled pilot trial. Am J Health Behav. 2016;40(4):503-13.

35. Rossom RC, Solberg LI, Magnan S, et al. Impact of a national collaborative care initiative for patients with depression and diabetes or cardiovascular disease. Gen Hosp Psychiatry. 2017;44:77-85.
36. Gonzalez JS, Fisher L, Polonsky WH. Depression in diabetes: have we been missing something important? Diabetes Care. 2011;34(1):236-9.

37. American Psychiatric Association. Diagnostic and statistical manual of mental disorder, 5th ed. Arlington: APA; 2013. 